\title{
PREVALENCE OF EXTENDED-SPECTRUM BETA-LACTAMASE PRODUCING ENTEROBACTERIACEAE MEMBERS ISOLATED FROM CLINICALLY SUSPECTED PATIENTS
}

\section{MOORTHY KANNAIYAN ${ }^{1,2}$, GEDIF MESERET ABEBE ${ }^{2}$, CHINNASAMY KANIMOZHI $^{1}$, PUNITHA THAMBIDURAI ${ }^{3}$, SARANYA ASHOKAPURAM SELVAM ${ }^{1}$, RAJA VINODHINI ${ }^{4 *}$, MICKYMARAY SURESH ${ }^{5}$}

\begin{abstract}
${ }^{1}$ Department of Microbiology, Vivekanandha College of Arts and Sciences for Women (Autonomous), Elayampalayam, Tiruchengode, Namakkal - 637 205, Tamil Nadu, India. ${ }^{2}$ Department of Microbiology, Wolaita Sodo University, Wolaita Zone, Ethiopia, Eastern Africa. ${ }^{3}$ Department of Microbiology, San International College of Arts and Science, Mavuthampathy Village, (Walayar), Coimbatore - 641 105, Tamil Nadu, India. ${ }^{4}$ Department of Microbiology, Shri Sakthikailaassh Women's College, Salem - 636 003, Tamil Nadu, India. ${ }^{5}$ Department of Biology, Central Bioscience Research Laboratories (CBRL) College of Science, Al- Zulfi, Majmaah University-Majmaah 11952, Kingdom of Saudi Arabia. Email: rvinodhini71@gmail.com
\end{abstract}

Received: 24 December 2017, Revised and Accepted: 30 January 2018

ABSTRACT

Objective: Emergence of extended-spectrum beta-lactamases (ESBLs) production poses another clinical problem with Gram-negative bacterial infections. The present study was aimed to evaluate the ESBL producers among various clinical samples of clinically suspected patients.

Methods: A total of 1279 samples (urine [918], pus [207] and stool [154]) were collected and 465 isolates (Escherichia coli [320], Enterobacter aerogenes [119] and Klebsiella pneumoniae [26]) were isolated and screened for the presence of ESBL producers using combination disc method and double disc synergy test.

Results: Of the 465 culture positive isolates, 130 (E. coli 93 [29.06\%], E. aerogenes 35 [29.41\%] and K. pneumoniae 2 [7.69\%]) were identified as ESBL producers. Among the three Enterobacteriaceae members, E. coli 93 (29.06\%) was found to be predominant ESBL producer next in order E. aerogenes $35(29.41 \%)$ and K. pneumoniae 2 (7.69\%). Maximum number of ESBL producers were recovered from urine $(\mathrm{n}=111)$ followed by pus ( $\mathrm{n}=14)$ and stool $(n=5)$. All the ESBL-producing isolates were subjected to antibiotic sensitivity test using 10 different antibiotics. ESBL producers were chiefly resistance to ceftriaxone followed by ceftazidime and cefotaxime. Of 130 ESBL producers, 15 (E. coli (8), E. aerogenes (6) and K. pneumoniae (1)] strains were selected for genotypic identification. Among, only two strains of E. aerogenes were positive isolates for CTX-M type ESBL in polymerase chain reaction.

Conclusion: This study concluded that among Enterobacteriaceae members, E. coli was the predominant ESBL producers and urine was noted as the prime source for the ESBL positive isolates when compared to other source. Genotypic identification was the best method to differentiate ESBL types which were essential to provide proper treatment.

Keywords: Extended-spectrum beta-lactamase, Enterobacteriaceae, Escherichia coli, Enterobacter aerogenes and Klebsiella pneumoniae.

(C) 2018 The Authors. Published by Innovare Academic Sciences Pvt Ltd. This is an open access article under the CC BY license (http://creativecommons. org/licenses/by/4. 0/) DOI: http://dx.doi.org/10.22159/ajpcr.2018.v11i5.19363

\section{INTRODUCTION}

Infections caused by extended-spectrum beta-lactamase (ESBL)producing, Gram-negative bacteria are associated with increased morbidity and mortality, which is linked to inappropriate or delayed antimicrobial treatment [1]. Since the introduction to the extended spectrum cephalosporins into clinical use, strains expressing ESBL have been reported from to the world in increasing numbers [2]. There is no consensus on the precise definition of ESBLs. A commonly used working definition is that the ESBLs are $\beta$-lactamases capable of hydrolysis of the antibiotics such as penicillins, first-, second- and third-generation cephalosporins and aztreonam (AT) (but not the cefamycins or carbapenems) and which are inhibited by $\beta$-lactamase inhibitors such as clavulanic acid [3]. The first report on plasmid-encoded $\beta$-lactamases capable of hydrolyzing the extended-spectrum cephalosporins was published in 1983 [4]. Among the family Enterobacteriaceae, the production of plasmid-mediated extended-spectrum $\beta$-lactamase (ESBLs) has emerged as an important mechanism of resistance to $\beta$-lactam drugs [5]. ESBLs have been found mainly in Klebsiella spp., and Escherichia coli, but have been also reported on other genera worldwide, such as Citrobacter, Enterobacter, Morganella, Proteus, Providencia, Salmonella, Serrati and P. seudomonas [6,7].

The ESBL genes are mostly plasmid-encoded [8], and most ESBLs can be divided into three genotypes: Temoniera (TEM), sulfhydryl variable (SHV), and CTX-M [3]. The predominant ESBL genotypes were TEM and SHV [9]. Most ESBLs are TEM and SHV enzyme derivatives characterized using a few point mutations at selected loci within the gene $[2,10]$. This enzyme was found in a blood culture isolate of $E$. coli from a Greek patient named TEM, hence the designation TEM [11]. The SHV-type ESBLs may be more frequently found in clinical isolates than any other type of ESBLs [12]. SHV refers to SHV. In addition, a genotype the CTX-M enzyme emerged worldwide when compared to TEM and SHV [9]. The plasmid-mediated ESBLs, which preferentially hydrolyze cefotaxime (CE) and are better inhibited by tazobactam than by sulbactam and clavulanate so-called as CTX-M enzymes [11]. In the 1990s, a novel type of ESBL, the CTX-M enzyme, emerged worldwide [9]. The CTX-M types, now exceeding 50 different types, can be divided into five groups based on their amino acid identities: CTX-M-1, CTX-M-2, CTX-M-8, CTX-M-9, and CTX-M-25 [13]. These enzymes are not very closely related to TEM and SHV $\beta$-lactamases as they show only $40 \%$ identity with these enzymes [14].

Infections caused by ESBL-producing bacteria often involve immunecompromised patients, making it difficult to eradicate these organisms in high-risk wards, such as intensive care units $[15,16]$. Drug susceptibility data are of major importance to the clinical management of patients infected by these organisms [10]. Thus, monitoring of the prevalence and the types of extended-spectrum $\beta$-Lactamase enzymes 
may contribute to defining the degree of the problem with a specific geographical area, and to establish a proper treatment protocol [17]. The aim of this study was to determine the prevalence of ESBL-producing Enterobacteriaceae members of the Department of Microbiology, DDC laboratory in Tiruchirappalli, India.

\section{MATERIALS AND METHODS}

The study was conducted in the Doctor's Diagnostic Centre, Tiruchirappalli, over a period of month from July to August 2013. A total of 1,279 consecutive, non-repetitive, Gram-negative isolates from various clinical samples such as urine $(n=918)$, pus $(n=207)$, and stool $(n=154)$ were included in the study.

\section{Isolation and identification}

The cultures were isolated from selective agar, MacConkey agar, and blood agar (Himedia, Mumbai) to study their cultural characteristics. A single isolated colony was considered for further studies and identification was done using the standard procedure. Gram's staining, morphological, cultural, and biochemical test were performed [18].

\section{Antibiotic sensitivity test}

The resistance to one or more $3^{\text {rd }}$ generation cephalosporin's (ceftazidime [CAZ], ceftriaxone (CTR), cefotaxime, etc.,) prompted us to detect ESBL producers, the common mechanism of beta-lactam resistance.

All the isolates were subjected to antimicrobial susceptibility agents and were determined using Disc Diffusion method of Kirby-Bauer [19] on Mueller-Hinton Agar as described by the Clinical and Laboratory Standard Institute (CLSI).The antibiotic discs used (HiMedia, Mumbai) were Cefoxitin (30 mcg), Cefepime (30 mcg), Cefepime+Tazobactam $(30 / 10 \mathrm{mcg})$, CAZ (30 mcg), ceftazidime+tazobactam (30/10 mcg), CTR (30 mcg), Cephotaxime or CE (30 mcg), cephotaxime+clavulanic acid (30/10 mcg), imipenem (10 mcg), and AT (30 mcg) [20].

\section{Combination disc method}

The combination disc test using both $\mathrm{CE}$ and CAZ, alone and in combination with clavulanic acid, was performed for the detection of ESBL according to the CLSI guidelines. In this test, an overnight culture suspension of the test isolate which was adjusted to 0.5 McFarland standards was inoculated using sterile cotton swab on the surface of a Mueller-Hinton agar plate. The CE $(30 \mu \mathrm{g})$ and cefotaxime-clavulanic acid $(30 / 10 \mu \mathrm{g})$ disks were placed $20 \mathrm{~mm}$ apart on the agar. Similarly, CAZ $(30 \mu \mathrm{g})$ and ceftazidime-clavulanic acid $(30 \mu \mathrm{g} / 10 \mu \mathrm{g})$ disks were placed $20 \mathrm{~mm}$ apart. After incubating overnight at $37^{\circ} \mathrm{C}, \geq 5 \mathrm{~mm}$ increase from the zone diameter for either antimicrobial agent which was tested in combination with clavulanic acid. Its zone, when tested for, was interpreted as positive for ESBL production [21].

\section{Double disc synergy test}

The test inoculums (0.5 McFarland tube) was spread as a lawn onto Mueller-Hinton agar (MHA) plate using a sterile cotton swab. A disc of CAZ $(30 \mu \mathrm{g})+$ clavulanic acid $(10 \mu \mathrm{g})$ was placed on the surface of MHA, then the disc of CAZ $(30 \mu \mathrm{g})$ was placed at the distance of $15 \mathrm{~mm}$ from the ceftazidime+clavulanic acid disc by the edge to edge. The inoculated plates were incubated at $35^{\circ} \mathrm{C}$ in the incubator for $18-24 \mathrm{~h}$. The zone of inhibition between the CAZ and ceftazidime+clavulanic acid was compared. The difference in the zone diameter of $\geq 5 \mathrm{~mm}$ was interpreted as positive for ESBL production [22].

\section{Molecular identification}

Isolation of DNA

TE buffer was added to overnight grown cells by gentle pipetting. Then, each of the tubes were added with $30 \mu \mathrm{l}$ of $10 \%$ SDS and $3 \mu \mathrm{lof} 20 \mathrm{mg} / \mathrm{ml}$ Proteinase $\mathrm{K}$. The tubes were vortexed and then incubated at $37^{\circ} \mathrm{C}$ for 1 h. A volume of $100 \mu \mathrm{l}$ of $\mathrm{CTAB} / \mathrm{NaCl}$ solution was added to $150 \mu \mathrm{l}$ of $5 \mathrm{M}$ $\mathrm{NaCl}$, mixed well and incubated at $65^{\circ} \mathrm{C}$ for $10 \mathrm{~min}$ and an equal volume of chloroform:Isoamyl alcohol mixture was added and centrifuged. The aqueous viscous supernatant was transferred to fresh tubes equal volumes of phenol:chloroform:isoamyl alcohol (25:24:4) was added. After centrifugation, to the supernatant ice-cold isopropanol was added and mixed well. The pellets were washed with $70 \%$ ethanol, and the nucleic acids were recovered by centrifugation. The pellets were then kept for drying after the complete removal of the supernatant and finally resuspended in $15 \mu \mathrm{l}$ of distilled water and stored at $4^{\circ} \mathrm{C}$ [23]. Polymerase chain reaction (PCR) amplification for CTX-M was carried out for all the isolates, based on the producers described by Woodford et al. [24]. After this, the amplified DNA fragments were purified from agarose gels using QIA gel extraction kit manufacturer's protocol and sequenced using forward and reverse about $771 \mathrm{bp}$ were carried out in Xcelris, Ahmedabad.

\section{RESULTS}

A total of 1279 various clinical samples were recovered from clinically suspected patients which include, urine 918 (71.77\%), pus 207 (16.18\%), and stool 154 (12.04\%). Based on the standard laboratory procedures, three Enterobacteriaceae members (E. coli 320 [68.81\%], Enterobacter. aerogenes 119 [25.59\%] and Klebsiella pneumoniae 26 [5.59\%]) are identified in Table 1.

Further, these 465 isolates were screened for the presence of ESBL producers using combination disc method and double disc synergy test. Of the 465 culture positive isolates, 130 (E. coli 93 [29.06\%], E. aerogenes 35 [29.41\%], and K. pneumoniae 2 [7.69\%]) were identified as ESBL producers, and remaining 335 were nonESBL producers (E. coli 93 [70.93\%], E. aerogenes 35 (70.58\%), and K. pneumoniae 2 (92.30\%)] (Table 2).

A maximum number of ESBL producers were recovered from urine $(n=111)$ followed by pus $(n=14)$ and stool $(n=5)$. Female patients were more prone to infection caused by ESBL producing isolates 78 (urine [38], pus [10], and stool [4]) than male patients 52 (urine [73], pus [4], and stool [1]) (Fig. 1).

The susceptibility profile of ESBL producers is depicted in Fig. 2. Of the 10 antibacterial agents, a maximum number of ESBL producers were resistance to CTR $(126 / 130)$ followed by CAZ $(115 / 130)$ and CE $(110 / 130)$. Interestingly, except one isolate $(1 / 130)$, all the ESBL producers were susceptible to imipenem.

For the genotypic identification, 15 ESBL positive strains (E. coli [8], E. aerogenes [6] and $K$. pneumoniae [1]) were selected, among two strains of E. aerogenes were positive isolates for CTX-M type ESBL in PCR. The amplified CTX-M gene after gel elution was sequenced using forward and reverse, about 415 and 771 bp were carried out in, Xcelris, Ahmedabad. The sequences obtained were aligned with previously published sequences available in NCBI using BLAST [25]. The gene accession number was KJ131192.1 and KJ131193.1. The sequence of the amplified product was as follows.

\section{KJ131192.1}

GTTCACGCTG ATGGCGACGG CAACCGTCAC GCTGTTGTTA GGAAGTGTGC CGCTGTATGC

GCAAACGGCG GACGTACAGC AAAAACTTGC CGAATTAGAG CGGCAGTCGG GAGGCAGACT

GGGTGTGGCA TTGATTAACA CAGCAGATAA TTCGCAAATA CTTTATCGTG CTGATGAGCG

CTTTGCGATG TGCAGCACCA GTAAAGTGAT GGCCGCGGCC GCGGTGCTGA AGAAAAGTGA

AAGCGAACCG AATCTGTTAA ATCAGCGAGT TGAGATCAAA AAATCTGACC TTGTTAACTA

TAATCCGATT GCGGAAAAGC ACGTCAATGG GACGATGTCA CTGGCTGAGC TTAGCGCGGC CGCGCTACAG TACAGCGATA ACGTG GCG 


\section{KJ131193.1}

CAGTTCACGC TGATGGCGAC GGCAACCGTC ACGCTGTTGT TAGGAAGTGT GCCGCTGTAT

GCGCAAACGG CGGACGTACA GCAAAAACTT GCCGAATTAG AGCGGCAGTC GGGAGGCAGA

CTGGGTGTGG CATTGATTAA CACAGCAGAT AATTCGCAAA TACTTTATCG TGCTGATGAG

CGCTTTGCGA TGTGCAGCAC CAGTAAAGTG ATGGCCGCGG CCGCGGTGCT GAAGAAAAGT

GAAAGCGAAC CGAATCTGTT AAATCAGCGA GTTGAGATCA AAAAATCTGA CCTTGTTAAC

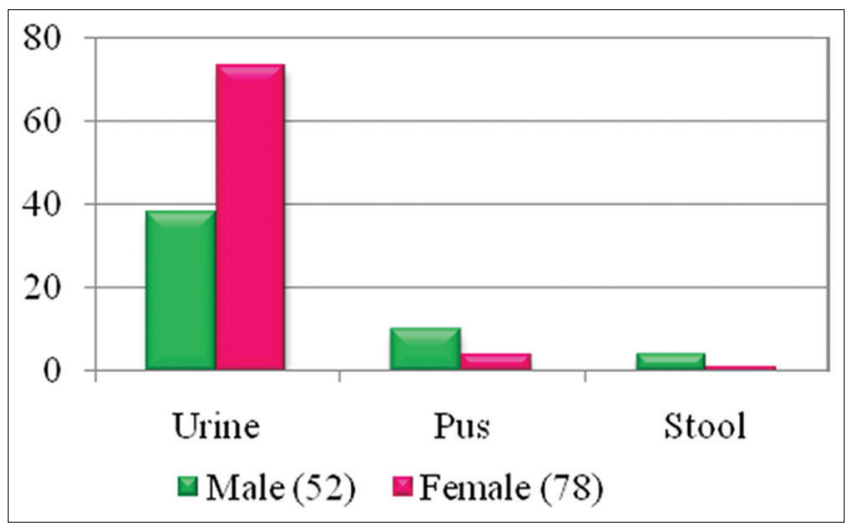

Fig. 1: Prevalence of extended-spectrum beta-lactamase over the sex distribution

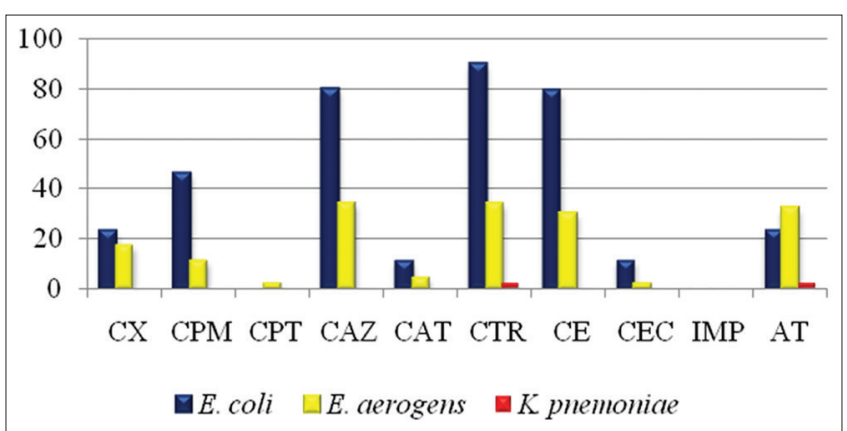

Fig. 2: Resistant patterns of extended-spectrum beta-lactamase producers from different sample

Table 1: Frequency of Gram-negative bacteria among various clinical samples

\begin{tabular}{lllll}
\hline Sample & \multicolumn{3}{l}{ Culture positive } & \\
\cline { 2 - 5 } & E. coli & E. aerogenes & K. pneumoniae & Total \\
\hline Urine $(\mathrm{n}=918)$ & 253 & 101 & 18 & 372 \\
Pus $(\mathrm{n}=207)$ & 34 & 18 & 8 & 60 \\
Stool $(\mathrm{n}=154)$ & 33 & - & - & 33 \\
Total & 320 & 119 & 26 & 465 \\
\hline
\end{tabular}

Escherichia coli: E. coli, Enterobacter aerogenes: E. aerogenes, Klebsiella pneumonia: K. pneumonia, ESBL: Extended-spectrum beta-lactamase
TATAATCCGA TTGCGGAAAA GCACGTCAAT GGGACGATGT CACTGGCTGA GCTTAGCGCG

GCCGCGCTAC AGTACAGCGA TAACGTGG

\section{DISCUSSION}

In hospital environment, resistance of bacterial pathogens to common antibiotic therapies was increasing at an alarming rate [26]. The use of antibiotics for any infection causes a "selective pressure" on bacterial populations, which emerge the resistant mutants and it can flourish [27]. The incidence of ESBL producing strains of clinical isolates has been steadily increasing from the past years resulting in limitation of therapeutic options [28]. These bacteria are showing rising rates of resistance to current therapies [29]. The problems which are associated with ESBLs include multidrug resistance, difficulty in detection and treatment, and increased mortality. ESBL-producing organisms, being the most common nosocomial pathogens, it is essential to detect and treat them as early as possible [30].

Nosocomial infections with Gram-negative bacilli are not uncommon for the local setting and can be perceived as a growing threat to public health [31]. In this study, out of 1279 different clinical samples, 465-Gramnegative isolates were recovered among that E. coli 320 (68.81\%), E. aerogenes 119 (25.59\%), and K. pneumoniae 26 (5.59\%). Similarly, a previous study recorded that, E. coli $(42.4 \%)$ was the most predominant isolate followed by K. pneumoniae (28.5\%) [32]. However, the study conducted by Nazneen et al. (47\%) [31], Menon et al. (47.14\%) [33], Shobha et al. (45.62\%) [34], and Nevine et al. (41.17\%) [35] recorded that K. pneumoniae was the predominant isolate.

The important reason for its detection is a failure to treat ESBL-producing organisms due to limited therapeutic choices [36]. The isolates resistant to cefotaxime were tested for ESBL production by double disc synergy test method [37-39]. Similarly, in the present study, ESBL production was detected using combination disc method and double disk synergy test. Similarly, a study conducted by Umadevi et al. [30] used the combination disc method, and some other studies used the double disk synergy test of the detection of ESBL producer [40-43].

ESBL-producing organisms predominantly isolated from urine sample [31,44-47]. Similarly, in our study, urine sample (85.38\%) was the major source of ESBL producing strains followed by pus (10.76\%) and stool (3.84\%). However, a study by Sharma et al. [41] recorded that high prevalence of ESBL producers we isolated from respiratory tract samples (63.83\%) was the major source of ESBL-producing strains followed by stool samples, urine, body fluid, pus, and blood.

The sex plays an important role in the infection which was affected by Gram-negative ESBL-producing organisms. A study by Shah et al. [48] was documented that male patients $10(62.50 \%)$ were predominant than female $6(37.50 \%)$ for the ESBL producers. Conversely, in our study, ESBL-producing isolates were predominantly recorded in female $(n=78)$ than male $(n=52)$. ESBL-producing isolates from urine were more common to males than females, in agreement with a previous report [49]. Contrary, in our study, male patients were more prevalent to ESBL in pus (10) and stool (5) samples than the female patients. On the other hand, ESBL prevalence in female patients was found to be the highest in urine samples (73).

Table 2: Detection of ESBL production in E. coli, E. aerogenes and K. pneumoniae

\begin{tabular}{|c|c|c|c|c|c|c|c|c|}
\hline \multirow[t]{2}{*}{ Sample } & \multicolumn{4}{|c|}{ ESBL producers } & \multicolumn{4}{|c|}{ NonESBL producers } \\
\hline & E. coli & E. aerogenes & K. pneumoniae & Total & E. coli & E. aerogenes & K. pneumoniae & Total \\
\hline Urine & 80 & 30 & 1 & 111 & 173 & 71 & 17 & 261 \\
\hline Pus & 8 & 5 & 1 & 14 & 26 & 13 & 7 & 46 \\
\hline Stool & 5 & - & - & 5 & 28 & - & - & 28 \\
\hline Total & 93 & 35 & 2 & 130 & 227 & 84 & 24 & 335 \\
\hline
\end{tabular}

Escherichia coli: E. coli, Enterobacter aerogenes: E. aerogenes, Klebsiella pneumonia: K. pneumonia, ESBL: Extended-spectrum beta-lactamase 
According to the geographical region, the fraction of putative ESBLproducing isolates can vary. These variations could be due to the differences in selecting a type of antibiotic, antibiotic selection pressure, local antibiotic, and prescribing habits, which differ from state to state, country to country, and from institution to institution. The prevalence of ESBL-producing bacteria has been on the rise, particularly in Asia compared to other regions [50]. A study from China, the figures of ESBL producers vary between 25 and $40 \%$ [51]. In India, the prevalence rate varies in different institutions from $28 \%$ to $84 \%$ [52], but Ali et al. [53] reported that ESBL producers making a frequency of $45 \%$. Another study recorded that ESBL production was found to be $52.49 \%$ [41]. Similarly, a study from Malaysia in 2001 by Nurul et al. [54] depicted a prevalence of ESBL as $58.6 \%$. A recent study in 2005, from New Delhi, showed $68.78 \%$ of the strains of Gram-negative bacteria to be ESBL producers [55]. A study by Nazneen et al. [31], Mathur et al. [56], and Nevine et al. [35] noted 61\%,68\%, and $65.8 \%$ of ESBL producer correspondingly, these prevalence were quite high when compared to our studies. Differently, in our study, the prevalence of ESBL producers was $27.95 \%$. A study by Basavaraj et al. [57] from Karnataka and Sharma and Grover [58] from Himachal-Pradesh reported an incidence of $32.1 \%$ and $38.5 \%$ which is slightly accordance with our results. While studies conducted by Rodrigues et al. [59], Kumar et al. [60], and Menon et al. [33] reported 6.9\%, 19.8\%, and 20\% respectively, which were quite low when compared to our study.

ESBLs in Gram-negative bacteria have emerged as a major problem of hospitalized as well as community-based patients [31]. Important ESBL-producing Gram-negative bacilli includes K. pneumoniae, E. coli, and P. mirabilis, Enterobacterspp., Citrobacter freundii, P. aeruginosa, Acinetobacter and Stenotrophomonas maltophilia [61]. A study from Egypt reported that $46 \%$ of ESBL-producing isolates of $K$. pneumoniae were from the clinical isolates [62]. ESBL are more prevalent in Klebsiella spp. followed by E. coli [63]. Similarly, a study by Nazneen et al. [31], Mathur et al. [56], Gupta et al. [64], Sharma et al. [41], and Ali et al. [53], were reported that, $K$. pneumoniae $74 \%, 73 \%, 71 \%, 67 \%$, and $57 \%$ was more prevalent than $E$. coli $62 \%, 62 \%, 64 \%, 57 \%$, and $39.1 \%$, respectively. Conversely, in the present study, the highest incidence of ESBL was noted in E. coli (29.06\%), E. aerogenes (29.41\%), and K. pneumoniae (7.69\%).

Klebsiella is the genus which frequently harbors ESBL [65]. However, in other studies, E. coli was the major ESBL producer $[30,44,66,67]$. Similarly, in the present study, the highest incidence of ESBL was noted in E. coli $(29.06 \%)$, E. aerogenes $(29.41 \%)$ and $K$. pneumoniae (7.69\%). Following studies were also reported that E. coli was the predominant ESBL producers. A study reported that $81 \%$ of the $E$. coli and $74 \%$ of the K. pneumoniae isolates were ESBL producers [30]. In the same way, a study noted that the highest ESBL producer was $E$. coli (64.2\%) followed by K. pneumoniae (60.1\%) [32]. Another study from southern India reported an incidence of $58.06 \%$ for ESBL producing E. coli and $57.14 \%$ for ESBL-producing Enterobacter spp. [68]. A similar prevalence was observed that $55.69 \%(\mathrm{n}=93)$ were E. coli and $44.31 \%$ $(\mathrm{n}=74)$ were K. pneumoniae isolates were ESBL producer [69]. Shrestha et al. [70] reported that the prevalence of ESBL-producing organisms was found to be 54 (18\%); among which E. coli was 29 (53.7\%), K. pneumoniae (14.8\%). A study by Jain et al. reported that maximum ESBL producers were found among E. coli isolates $(80.9 \%)$ followed by K. pneumoniae (75\%) [71]. A report from Coimbatore (India) showed that ESBL production was $41 \%$ in E. coli and $40 \%$ in K. pneumoniae [72]. Kulkarni et al. [47] in their study reported that E. coli (40.7\%) was the most frequent and K. pneumoniae (15.9\%).

\section{CONCLUSION}

The study results suggested that, among Enterobacteriaceae members, E. coli was the predominant ESBL producers and urine was noted as the prime source for the ESBL positive isolates when compared to another source. Although many phenotypic methods were available, genotypic identification was the best method to differentiate ESBL types which were essential to provide proper treatment. Constant and careful surveillance, proper detection methods, and proper management are recommended to control the spread of these organisms as the infections by ESBL-producing organisms.

\section{ACKNOWLEDGMENTS}

The authors are thankful to Prof. M. Karunanithi, Chairman and Secretary, Vivekanandha Educational Institutions, Elayampalayam, and Dr. A. Malarvizhi, Head Department of Microbiology, Vivekanandha College of Arts and Sciences for Women (Autonomous), Elayampalayam, Tiruchengode, Namakkal District, Tamil Nadu for providing all the facilities for our research work.

\section{CONFLICT OF INTEREST}

We declare that we have no conflict of interest.

\section{REFERENCES}

1. Knudsen JD, Andersen SE, Bispebjerg Intervention Group. A multidisciplinary intervention to reduce infections of ESBL-and ampC-producing, gram-negative bacteria at a university hospital. PLoS One 2014;9:e86457.

2. Jacoby GA, Medeiros AA. More extended spectrum $\beta$ lactamases. Antimicrob Agents Chemother 1991;35:1697-704.

3. Paterson DL, Bonomo RA. Extended-spectrum beta-lactamases: A clinical update. Clin Microbiol Rev 2005;18:657-86.

4. Knothe H, Shah P, Krcmery V, Antal M, Mitsuhashi S. Transferable resistance to cefotaxime, cefoxitin, cefamandole and cefuroxime in clinical isolates of Klebsiella pneumoniae and Serratia marcescens. Infection 1983;11:315-17.

5. Bonnet R, De Champs C, Sirot D, Chanal C, Labia R, Sirot J, et al. Diversity of TEM mutants in Proteus mirabilis. Antimicrob Agents Chemother 1999;43:2671-7.

6. Choi SH, Lee JE, Park SJ, Kim MN, Choo EJ, Kwak YG, et al. Prevalence, microbiology, and clinical characteristics of extendedspectrum beta-lactamase-producing Enterobacter spp., Serratia marcescens, Citrobacter freundii, and Morganella morganii in Korea. Eur J Clin Microbiol Infect Dis 2007;26:557-61.

7. Ivanova D, Markovska R, Hadjieva N, Schneider I, Mitov I, Bauernfeind A. Extended-spectrum $\beta$-lactamaseproducing Serratia marcescens outbreak in a Bulgarian hospital. J Hosp Infect 2008;70:60-5.

8. Nakamura T, Komatsu M, Yamasaki K, Fukuda S, Miyamoto Y, Higuchi $\mathrm{T}$, et al. Epidemiology of Escherichia coli, Klebsiella species, and Proteus mirabilis strains producing extended-spectrum $\beta$-lactamases from clinical samples in the Kinki region of Japan. Am J Clin Pathol 2012;137:620-6.

9. Bradford PA. Extended-spectrum beta-lactamases in the $21^{\text {st }}$ century: Characterization, epidemiology, and detection of this important resistance threat. Clin Microbiol Rev 2001;14:933-51.

10. Bush K, Jacoby GA, Medeiros AA. A functional classification scheme for $\beta$-lactamases and its correlation with molecular structure. Antimicrob Agents Chemother 1995;39:1211-33.

11. Giamarellou H. Multidrug resistance in gram-negative bacteria that produce extended-spectrum b-lactamases (ESBLs). Clin Microbiol Infect 2005;11 Suppl 4:1-16.

12. Jacoby GA. Extended-spectrum beta-lactamases and other enzymes providing resistance to oxyimino-beta-lactams. Infect Dis Clin North Am 1997; 11:875-87.

13. Bonnet R. Growing group of extended-spectrum $\beta$-lactamases: The CTX-M enzymes. Antimicrob Agents Chemother 2004;48:1-14.

14. Tzouvelekis LS, Tzelepi E, Tassios PT, Legakis NJ. CTX-mtype b-lactamases; An emerging group of extended-spectrum enzymes. Int J Antimicrob Agents 2000;14:137-43.

15. Bonnet R, Sampaio JL, Chanal C, Sirot D, De Champs C, Viallard JL, et al. A novel class a extended-spectrum beta-lactamase (BES-1) in Serratia marcescens isolated in Brazil. Antimicrob Agents Chemother 2000;44:3061-8.

16. Jean SS, Hsueh PR, Lee WS, Chang HT, Chou MY, Chen IS, et al. Nationwide surveillance of antimicrobial resistance among Enterobacteriaceae in intensive care units in Taiwan. Eur J Clin Microbiol Infect Dis 2009;28:215-20.

17. Batchoun RG, Swedan SF, Shurman AM. Extended spectrum $\beta$-lactamases among gram-negative bacterial isolates from clinical specimens in three major hospitals in Northern Jordan. Int J Microbio 2009. Article ID: 513874,9 pages. 
18. Collee JG, Fraser AG, Marmion BP, Simmons A. Mackie and McCartney Practical Medical Microbiology. $14^{\text {th }}$ ed. London: Churchill Livingstone; 1996. p. 417-23.

19. Bauer AW, Kirby MD, Sherris JC, Turck M. Antibiotic susceptibility testing by standardized single disc diffusion method. Am J Clin Pathol 1966;45:493-6.

20. Clinical and Laboratory Standards Institute (CLSI). Performance Standard for Antimicrobial Susceptibility Testing. $16^{\text {th }}$ Informational Supplement. Wayne, PA: CLSI Document M100-S16; 2006.

21. Clinical and Laboratory standards Institute (CLSI). Performance standard for Antimicrobial Susceptibility Testing. Wayne, PA. USA: CLSI Approved Standards CLSI M-100-S20; 2010.

22. Balan K. Detection of extended spectrum beta lactamase among gram negative clinical isolates from a tertiary care hospital in south India. Ind J Med Sci 2013;1:28-30.

23. Ansubel M, Brent R, Kingston RE, Moore DD, Seidman JG, Smith JA, et al. Current Protocols in Molecular Biology. Vol. 1. New York, USA: Wiley \& Sons, Inc.; 1995.

24. Woodford N, Fagan EJ, Ellington MJ. Multiplex PCR for rapid detection of genes encoding CTX-M extended-spectrum (beta)-lactamases. J Antimicrob Chemother 2006;57:154-5.

25. Altschul SF, Madden TL, Schäffer AA, Zhang J, Zhang Z, Miller W, et al. Gapped BLAST and PSI-BLAST: A new generation of protein database search programs. Nucleic Acids Res 1997;25:3389-402.

26. Yusuf I, Haruna M, Yahaya H. Prevalence and antibiotic susceptibility of Ampc and ESBL producing clinical isolates at a tertiary health care center in Kano, Northwest Nigeria. Afr J Cln Exper Microbiol 2013;2:109-19.

27. Patel MH, Trivedi GR, Patel SM, Vegad MM. Antibiotic susceptibility pattern in urinary isolates of gram negative bacilli with special reference to ampC $\beta$-lactamase in a tertiary care hospital. Urol Ann 2010;2:7-11.

28. Podschun R, Ullmann U. Klebsiella spp. As nosocomial pathogens: Epidemiology, taxonomy, typing methods, and pathogenicity factors. Clin Microbiol Rev 1998;11:589-603.

29. Soltani R, Ehsanpoor M, Khorvash F, Shokri MM. Antimicrobial susceptibility pattern of extended-spectrum $\beta$-lactamase-producing bacteria causing nosocomial urinary tract infections in an Iranian referral teaching hospital. J Res Pharm Pract 2014;3:6-11

30. Umadevi S, Kandhakumari G, Joseph NM, Kumar S, Easow JM, Stephen S, et al. Prevalence and antimicrobial susceptibility pattern of ESBL producing gram negative bacilli. J Clin Diagn Res 2011;5:236-9.

31. Nazneen S, Jayshree B, Ajit D, Jyoti B. Prevalence of extended spectrum beta lactamase (ESBL) producing gram negative bacilli from various clinical isolates. IOSR J Dent and Med Sci 2014;13:8-11.

32. Chaudhary M, Kumar S, Payasi A. Prevalence and antimicrobial sensitivity of extended-spectrum $\beta$-lactamase producing gram negative bacteria from clinical settings in India from 2010-2012. Int J Med and Med Sci 2013;46:46-9.

33. Menon T, Bindu D, Kumar CP, Nalini S, Thirunarayan MA. Comparison of double disc and three dimensional methods to screen for ESBL producers in a tertiary care hospital. Indian J Med Microbiol 2006;24:117-20.

34. Shobha KL, Ramachandra L, Rao G, Majumder S, Rao SP. Extended spectrum beta-lactamases (ESBL) in gram negative bacilli at a tertiary care hospital. J Diagn Clin Res 2009;3:1307-12.

35. Nevine S, Fam M, El-Damarawy M. CTX-M-15 extended-spectrum beta-lactamases detected from intensive care unit of an Egyptian medical research institute. Res J Med Med Sci 2008;3:84-91.

36. Ullah F, Malik SA, Ahmed J. Antimicrobial susceptibility pattern and ESBL prevalence in Klebsiella pneumonia from urinary tract infection in the North-West of Pakistan. Afr J Microbiol Res 2009;3:676-80.

37. Tankhiwale SS, Jalgaonkar SV, Ahamad S, Hassani U. Evaluation of extended spectrum beta lactamase in urinary isolates. Indian J Med Res 2004;120:553-6.

38. Yusuf I, Arzai AH, Umar A, Magaji N, Salisu N, Tukur A, et al. Prevalence of extended spectrum beta lactamases (ESBL) producing Escherichia coli and Klebsiella pneumoniae in tuberculosis patients in Kano, Nigeria. Bajopas 2011;4:1

39. Raja MM, Ahmed JS. Evaluation of extended spectrum of beta lactamases producing Escherichia coli isolated from hospitalized patients M. Int J Adv Res 2013;19:309-15.

40. Jain A, Roy I, Gupta MK, Mala K, Agarwal SK. Prevalence of extended-spectrum beta-lactamase producing Gram-negative bacteria in septicaemic neonates in a tertiary care hospital. J Med Microb 2003;52:421-5.

41. Sharma M, Pathak S, Preeti S. Prevalence and antibiogram of extended spectrum $\beta$-lactamase (ESBL) producing gram negative bacilli and further molecular characterization of ESBL producing Escherichia coli and Klebsiella spp. J Clin Diag Res 2013;7:2173-7.

42. Hansotia JB, Agarwal V, Pathak AA, Saoji AM. Extended spectrum beta-lactamase mediated resistance to third generation cephalosporins in Klebsiella pneumoniae in Nagpur, central India. Indian J Med Res 1997;105:158-61.

43. Dechen CT, Shyamasree D, Luna A, Ranabir P, Takhellambam SK. Extended spectrum beta-lactamase detection in gram-negative bacilli of nosocomial origin. J Glob Infect Dis 2009;1:87-92.

44. Shanthi M, Sekar U. Extended spectrum beta lactamase producing Escherichia coli and Klebsiella pneumoniae: Risk factors for infection and impact of resistance on outcomes. J Assoc Physicians India 2010;58 Suppl:41-4.

45. Iraj A, Nilufar YN. Antibiogram of extended spectrum beta-lactamase (ESBL) producing Escherichia coli and Klebsiella pneumoniae isolated from hospital samples. Bangladesh J Med Microbiol 2010;4:32-6.

46. Saba R, Muhammad F, Shahida H. Prevalence and comparison of betalactamase producing Escherichia coli and Klebsiella spp. from clinical and environmental sources in Lahore, Pakistan. Af J Microbio Res 2012;6:465-70.

47. Kulkarni R, Vaishali D, Ghadge D, Bhore A. A study of extended spectrum beta lactamases (ESBL) producers in clinical isolates. Med J West India 2013;41:18-22.

48. Shah RK, Singh YI, Sanjana RK, Navin C, Dominic S. Study of extended spectrum beta-lactamases (ESBLs) producing Klebsiella species in various clinical specimens: A preliminary report. J Coll Med Sci Nepal 2010;6:19-23.

49. Graffunder EM, Preston KE, Evans AM, Venezia A. Risk factors associated with extended-spectrum $\beta$-lactamase-producing organisms at a tertiary care hospital. J Antimicro Chemother 2005;56:139-45.

50. Harada Y, Morinaga Y, Yamada K, Migiyama Y, Nagaoka K, Nakamura U, et al. Clinical and molecular epidemiology of extendedspectrum $\beta$-lactamase-producing Klebsiella pneumoniae and Escherichia coli in a Japanese tertiary hospital. J Med Microb Diagn 2013;2:2161-70.

51. Yu Y, Zhou W, Chen Y, Ding Y, Ma Y. Epidemiological and antibiotic resistant study on extended-spectrum beta lactamase-producing Escherichia coli and Klebsiella pneumonia in Zhejiang province. Chin Med J (Engl) 2002;115:1479-82.

52. Das A, Ray P, Garg R, Kaur B. Proceedings of the Silver Jubilee Conference. New Delhi: All India Institute of Medical Sciences; Extended Spectrum Beta-Lactamase Production in Gram Negative Bacterial Isolates from Cases of Septicemia; 2001.

53. Ali AM, Rafi S, Qureshi AH. Frequency of Extended Spectrum Beta Lactamase Producing Gram Negative Bacilli among Clinical Isolates at Clinical Laboratories of Army Medical College, Rawalpindi. Available from: http://www.ayubmed.edu.pk/JAMC/PAST/16-1/Aarif.htm.

54. Nurul MA, Loo HK, Subramaniam G, Wong EH, Selvi P, Ho S, et al. Faecal prevalence of extended-spectrum ß-lactamase (ESBL)producing coliforms in a geriatric population and among haematology patients. Malaysian J Pathol 2005;27:75-81

55. Mohanty S, Singhal R, Sood S, Dhawan B, Das BK, Kapil A, et al. Comparative in vitro activity of beta-lactam/beta-lactamase inhibitor combinations against gram negative bacteria. Indian $\mathrm{J}$ Med Res 2005; $122: 425-8$

56. Mathur P, Kapil A, Das B, Dhawan B. Prevalence of extended spectrum beta lactamase producing gram negative bacteria in a tertiary care hospital. Indian J Med Res 2002;115:153-7.

57. Basavaraj PJ, Basavaraj PV. The prevalence of ESBL among Enterobacteriaceae in a tertiary care hospital of north Karnataka, India. J Clin Diag Res 2011;5:470-5.

58. Sharma A, Grover PS. Application of WHONET for the surveillance of antimicrobial resistance. Indian J Med Microbiol 2004;22:115-8.

59. Rodrigues C, Joshi P, Jani SH, Alphonse M, Radhakrishnan R, Mehta A. Detection Of $\beta$-lactamases in nosocomial gram negative clinical isolates. Indian J Med Microbiol 2004;22:247-50.

60. Kumar MS, Lakshmi V, Rajagopalan R. Occurrence of extended spectrum beta-lactamases among Enterobacteriaceae spp. Isolated at a tertiary care institute. Indian J Med Microbiol 2006;24:208-11.

61. Coudron PE, Moland ES, Sanders CC. Occurrence and detection of extended-spectrum ß-lactamases in members of the family Enterobacteriaceae at a veterans medical center: Seek and you may find. J Clin Microbiol 1997;35:2593-7.

62. El-mahdy TS, Abdelaziz MO, El-domany RA. Prevalence and molecular characterization of extended spectrum $\beta$-lactamases in Klebsiella pneumoniae isolates from cancer patients and others. Int $\mathrm{J}$ Pharm Pharm Sci 2015;7:122-12. 
63. Nathisuwan S, Burgess DS, Lewis JS 2 ${ }^{\text {nd }}$. ESBLs: Epidemiology, detection and treatment. Pharmacotherapy 2001;28:920-8.

64. Gupta V, Singla N, Jagdish C. Detection of sells using third and fourth generation cephalosporins in double disc synergy test. Indian J Med Res 2007;126:486-7.

65. Khurana S, Taneja N, Sharma M. Extended spectrum $\beta$-lactamase mediated resistance in urinary tract isolates of family Enterobacteriaceae. Indian J Med Res 2002;116:145-9.

66. Abhilash KP, Veeraraghavan B, Abraham OC. Epidemiology and outcome of bacteremia caused by extended spectrum beta-lactamase (ESBL)-producing Escherichia coli and Klebsiella spp. In a tertiary care teaching hospital in south India. J Assoc Physicians India 2010;58 Suppl:13-7.

67. Wattal C, Goel N, Oberoi JK, Raveendran RS, Datta S, Prasad KJ. Surveillance of multidrug resistant organisms in a tertiary care hospital in Delhi, India. J Assoc Physicians India 2010;58 Suppl:32-6.

68. Ananthakrishnan AN, Kanungo R, Kumar A, Badrinath S. Detection of extended spectrum beta-lactamase producers among surgical wound infections and burn patients in JIPMER. Indian J Med Microbiol 2000;18:160-5.

69. Shaikh S, Fatima J, Shakil S, Rizvi MS, Kamal MA. Risk factors for acquisition of extended spectrum beta lactamases producing Escherichia coli and Klebsiella pneumonia in North-Indian hospitals. Saudi J Biol Sci 2015;22:37-41.

70. Shrestha S, Amatya R, Dutta R. Prevalence of extended spectrum beta lactamase $(\mathrm{ESBL})$ production in gram negative isolates from pyogenic infection in tertiary care hospital of Eastern Nepal. Nepal Med Coll J 2011;13:186-9.

71. Jain S, Geeta W, Rubina M. Prevalence and antimicrobial susceptibility pattern of ESBL producing gram negative bacilli in 200 cases of urinary tract infections. Int J Pharm Pharm Sci 2014;6:210-1.

72. Babypadmini S, Appalaraju B. Extended spectrum beta-lactamases in urinary isolates of Escherichia coli and Klebsiella pneumoniaeprevalence and susceptibility pattern in a tertiary care hospital. Indian J Med Microbiol 2004;22:172-4. 